The following resources related to this article are available online at www.sciencemag.org (this information is current as of November 12, 2009 ):

Updated information and services, including high-resolution figures, can be found in the online version of this article at:

http://www.sciencemag.org/cgi/content/full/326/5955/974

Supporting Online Material can be found at:

http://www.sciencemag.org/cgi/content/full/326/5955/974/DC1

This article cites $\mathbf{2 0}$ articles, 1 of which can be accessed for free:

http://www.sciencemag.org/cgi/content/full/326/5955/974\#otherarticles

This article appears in the following subject collections:

Physics

http://www.sciencemag.org/cgi/collection/physics

Information about obtaining reprints of this article or about obtaining permission to reproduce this article in whole or in part can be found at:

http://www.sciencemag.org/about/permissions.dtl 


\section{Observation of Half-Quantum Vortices in an Exciton-Polariton Condensate}

\author{
K. G. Lagoudakis, ${ }^{1 *}$ T. Ostatnický, ${ }^{2}$ A. V. Kavokin, ${ }^{2,3}$ Y. G. Rubo, ${ }^{4}$ R. André, ${ }^{5}$ B. Deveaud-Plédran ${ }^{1}$
}

Singly quantized vortices have already been observed in many systems, including the superfluid helium, Bose-Einstein condensates of dilute atomic gases, and condensates of exciton-polaritons in the solid state. Two-dimensional superfluids carrying spin are expected to demonstrate a different type of elementary excitations referred to as half-quantum vortices, characterized by a $\pi$ rotation of the phase and a $\pi$ rotation of the polarization vector when circumventing the vortex core. We detect half-quantum vortices in an exciton-polariton condensate by means of polarization-resolved interferometry, real-space spectroscopy, and phase imaging. Half-quantum vortices coexist with single-quantum vortices in our sample.

$\mathrm{Q}$ uantized vorticity is a property of quantum fluids that has been widely studied in various types of superfluids, either with or without spin $(1-4)$. Superfluids with a two-component (spinor) order parameter are characterized by a different type of vortices than those found in conventional scalar superfluids $(5-8)$. This new type of vortices is the socalled half-quantum vortices (HQV). They have lower energy with respect to full vortices and constitute the elementary excitations of spinor superfluids. When circumventing their core, the phase and the polarization vector experience $\mathrm{a} \pm \pi$ rotation. In this sense, HQV can be understood as a half-phase vortex combined with a halfpolarization vortex (5). In ${ }^{4} \mathrm{He}$ superfluids, the HQV cannot be formed because the spin degree of freedom is absent. However, in ${ }^{3} \mathrm{He}(5,6)$ or in triplet superconductors $(7,8)$, the order parameter has two or more components, so the formation of HQV is possible. So far, experiments have not presented unambiguous evidence for HQV in ${ }^{3} \mathrm{He}(9)$, although more reliable indications of the existence of HQV have been reported in cuprate superconductors $(10,11)$. Recently, HQV have been proposed as a smoking gun for the superfluid of exciton-polaritons in semiconductor microcavities (12). We report on a direct observation of HQVs in a high-temperature quantum fluid: microcavity exciton-polaritons. Exciton-polaritons are composite bosons carrying spin. They can occur in semiconductor microcavities in the strong coupling regime and are partly excitons and partly photons. The energy, wave vector, polarization, and statistics of cavity polaritons are directly transferred to light emitted by the microcavity due to photon tunneling through the mirrors of the cavity (13). Because of their photonic component, the cavity polaritons have an extremely low

${ }^{1}$ Institut de Photique et d'Electronique Quantique (IPEQ), École Polytechnique Fédérale de Lausanne (EPFL), 1015 Lausanne, Switzerland. ${ }^{2}$ School of Physics and Astronomy, University of Southampton, Highfield, Southampton S017 1B], UK. ${ }^{3}$ MarieCurie Chair of Excellence, University of Rome II, Via della Ricerca Scientifica 1, 00133 Rome, Italy. ${ }^{4}$ Centro de Investigación en Energía, Universidad Nacional Autónoma de México, Temixco, 62580 Morelos, Mexico. ${ }^{5}$ Institut Néel, Centre National de la Recherche Scientifique (CNRS), 38042 Grenoble, France.

*To whom correspondence should be addressed. E-mail: konstantinos.lagoudakis@epfl.ch effective mass on the order of $10^{-4}$ of the free electron mass. This allows for their Bose-Einstein condensation (BEC) at temperatures achievable
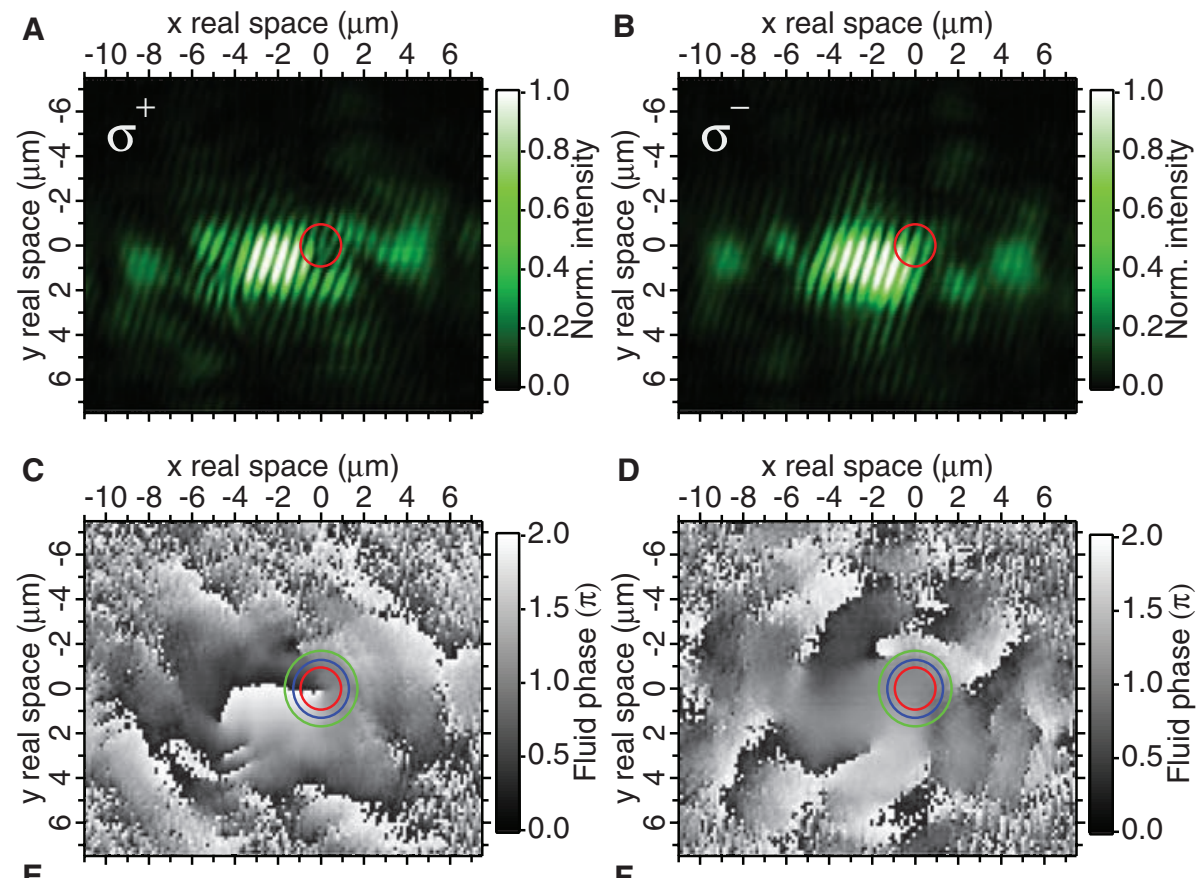

E
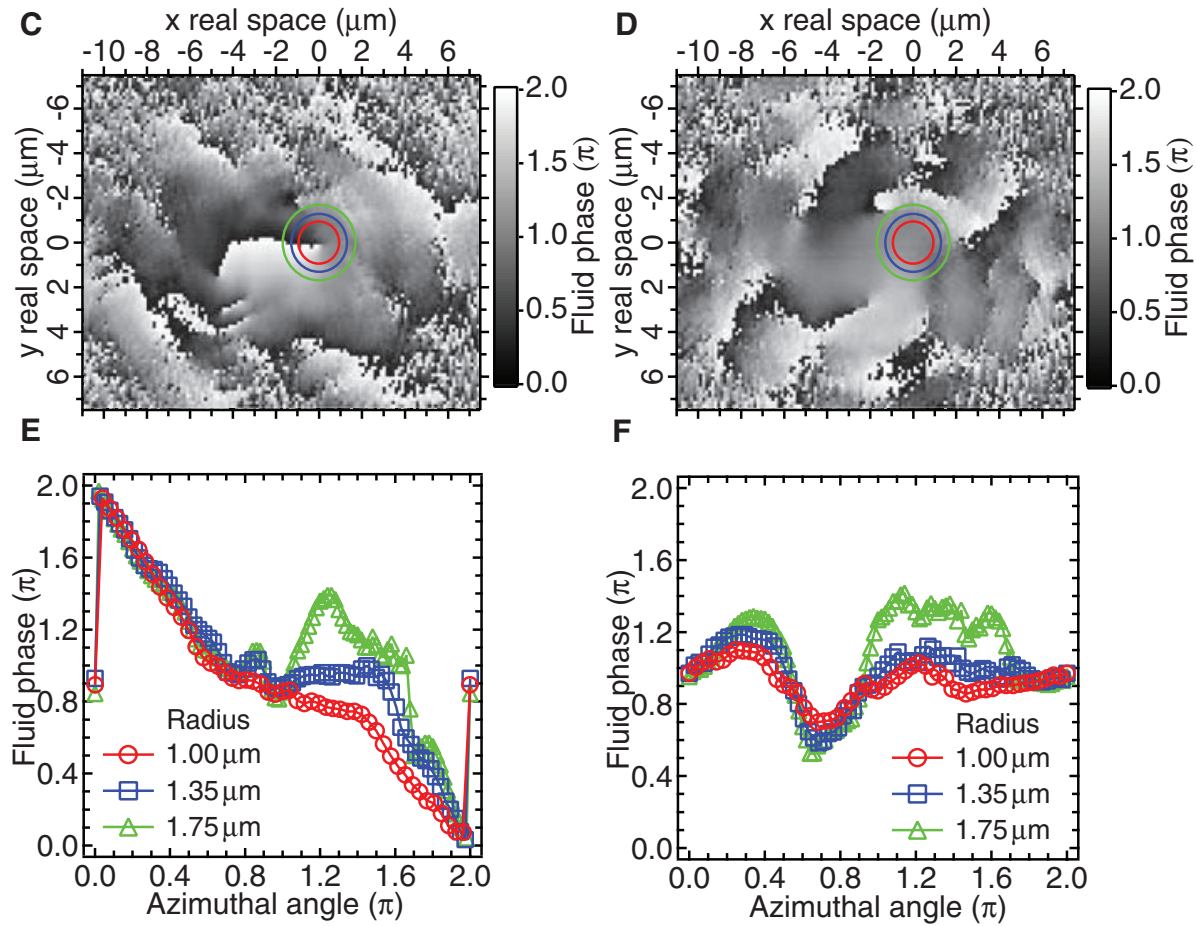

Fig. 1. (A and B) Reconstructed interferograms for $\sigma^{+}$and $\sigma^{-}$polarizations at the energy of the condensate. (C and D) Real space phase map extracted from the interferograms (A) and (B) for polarizations $\sigma^{+}$and $\sigma^{-}$, respectively. The three circles with different colors denote the real space paths over which we have plotted the phase as a function of the azimuthal angle in panels ( $\mathbf{E}$ and $\mathbf{F}$ ). The phase profiles in $\sigma^{+}$polarization (E) show that the phase changes by $-2 \pi$ when circumventing the vortex core, which is the signature of the singularity. In contrast, for the $\sigma^{-}$polarization (F), we see a quasi-flat phase profile with zero overall phase shift as one goes around the core. The farther we probe the phase away from the vortex core, the more the phase diverges from the linear behavior versus angle. 
polarization splitting in microcavities, which is responsible for the suppression of HQV in certain areas of the sample. Thus, in different parts of the sample the polariton fluid has a different topology.

To fully characterize a vortex in a polariton condensate, one needs two winding numbers, $(k, m)$, one for the polarization angle and one for the phase. One can express the order parameter of the condensate in the linear $x y$ basis as

$$
\psi_{l i n}(\boldsymbol{r})=\sqrt{n} e^{i \theta(\boldsymbol{r})}\left(\begin{array}{c}
\cos \eta(\boldsymbol{r}) \\
\sin \eta(\boldsymbol{r})
\end{array}\right)
$$

where $\theta(\mathbf{r})$ is the phase of the coherent polariton fluid and $\eta(\mathbf{r})$ is the polar angle that characterizes the orientation of the electric field of polaritons, i.e., the polarization angle. Vortices are described in this notation by rotation of the phase and the polarization as $\eta(\mathbf{r}) \rightarrow \eta(\mathbf{r})+2 \pi k$ and $\theta(r) \rightarrow \theta(r)+2 \pi m$ where $k, m$ can take integer or half-integer values with $(k+m) \in \mathbb{Z}$. Four types of half vortices are described by winding numbers $(k, m)=\left( \pm \frac{1}{2}, \pm \frac{1}{2}\right)$. To reveal the specific phenomenology of HQVs with respect to the integer vortices, it is convenient to analyze the circularly polarized components of the order parameter, which can be expressed as

$$
\begin{aligned}
& \psi_{\text {lin }}(\mathbf{r})= \\
& \frac{\sqrt{n}}{2}\left[e^{i(\theta(\mathbf{r})-\eta(\mathbf{r}))}\left(\begin{array}{c}
1 \\
i
\end{array}\right)+e^{i(\theta(\mathbf{r})+\eta(\mathbf{r}))}\left(\begin{array}{c}
1 \\
-i
\end{array}\right)\right]
\end{aligned}
$$

One can see that for $\eta(\mathbf{r}) \rightarrow \eta(\mathbf{r})+\pi$ and $\theta(\mathbf{r}) \rightarrow \theta(\mathbf{r})+\pi$ a zero rotation takes place for one circular polarization and a full $2 \pi$ rotation is achieved for the other circular polarization. This means that if one were to detect a half vortex, it would be easiest when looking in $\sigma^{+}$and $\sigma^{-}$ polarizations simultaneously. Then HQV would be observed as a full vortex in one polarization and no vortex in the other one. A signature for the phase vortex is a forklike dislocation in the interference pattern $(4,18)$. In the case of full phase vortices, the forklike dislocations are expected to be seen in the same place in both circular polarizations, whereas in the case of HQV the fork appears only in one of the circular polarizations. In the circular basis, one can write the
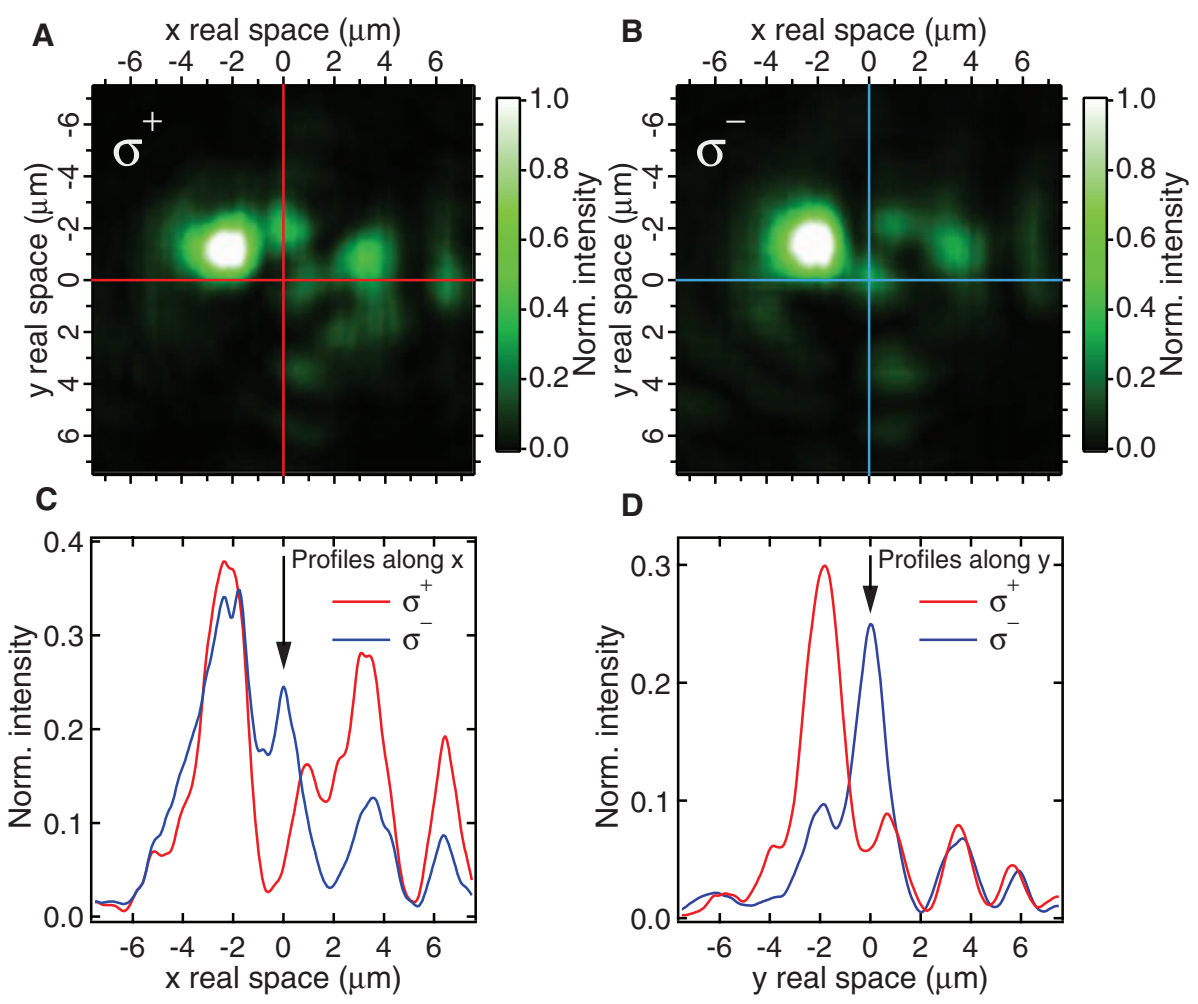

Fig. 2. (A and B) Polariton densities in real space at the frequency of the condensate for $\sigma^{+}$and $\sigma^{-}$ polarizations, respectively. It is easily seen that at the position of the vortex core $(0,0)$, which is indicated by the red cross for $\sigma^{+}$polarization and the blue cross for the $\sigma^{-}$polarization, there is a local minimum for $\sigma^{+}$polariton density and a local maximum for the $\sigma^{-}$density. (C) Density profiles along the $x$ direction for the two polarizations and (D) the corresponding density profiles along the $y$ direction. Experimentally measured polariton density behaves in excellent agreement with the theoretical prediction [Eq. 3 and (21)]. This behavior is also evident from the fact that half vortices must be fully circularly polarized at the centers of their cores. order parameter of HQV in cylindrical coordinates as

$$
\begin{aligned}
& \psi_{k, m}(\rho, \phi)= \\
& \sqrt{\frac{n}{2}} \mathrm{e}^{\mathrm{i} m \phi}\left\{\begin{array}{l}
{[f(\rho)+\operatorname{sgn}(k m) g(\rho)] \cdot \mathrm{e}^{-\mathrm{i} k \phi}} \\
{[f(\rho)-\operatorname{sgn}(k m) g(\rho)] \cdot \mathrm{e}^{\mathrm{i} k \phi}}
\end{array}\right\}
\end{aligned}
$$

with $\rho=\frac{r}{a}$ being the relative distance from the vortex core in vortex radii and $\phi$ being the angular coordinate. The form of the two radial density functions $f$ and $g$ is known (12) and will give zero density for one circular polarization $(f-g)$ and a finite density for the other polarization $(f+g)$, as it is expected for the simplistic image of a full vortex in one circular polarization and no singularities for the other circular polarization.

An important feature of polariton condensates is the presence of polarization splitting induced by the structural anisotropy and stationary disorder. This splitting pins the polarization vector of the condensate to a given crystal axis. It is theoretically predicted that HQVs still exist in this case but the spatial distribution of the polariton vector field is modified. Similarly to the vortices in multicomponent quantum Hall systems (19), the polariton half vortices acquire "strings" [or solitons; figure 16.1 in (6)], whereby the polarization angle rotates by $\pi(20)$. The width of the string is given by $\hbar / \sqrt{2 m^{*} \varepsilon}$, where $m^{*}$ is the effective mass of polaritons and $\varepsilon$ is the energy of the polarization splitting. HQVs remain the lowestenergy topological defects if this width is greater than or comparable to the excitation spot radius. However, when this length becomes comparable to the vortex core size $\left(a \approx \hbar / \sqrt{2 m^{*} \mu}\right.$, where $\mu$ is the chemical potential), the excitation of HQVs would require too much energy, and the integer phase vortices $(0, \pm 1)$ become elementary topological excitations. For a realistic vortex core size on the order of $\sim 2 \mu \mathrm{m}$ and the polariton mass $m^{*} \approx 10^{-4} m_{\mathrm{e}}$, pairs of HQVs will be replaced by integer phase vortices for polarization splittings $\varepsilon \geq 100 \mu \mathrm{eV}$. [Two close pairs of HQVs are shown in (21), figs. S2 and S3].

The situation in real microcavity samples is additionally complicated by the fact that the polarization splitting $\varepsilon$ fluctuates as a function of the coordinate in the plane of the cavity. This is why the HQV and integer phase vortices may coexist within the same condensate. The underlying mechanisms for the polarization splitting are thought to be the different penetration depths in the distributed Bragg reflectors (microcavity mirrors) for transverse electric and transverse magnetic polarizations (22) and the intrinsic anisotropy of the microcavity $(23,24)$. The anisotropy is expected to be the product of a number of parameters, including the alloy concentrations, the wedge, quantum well width fluctuations, and the built-in strain. Splittings vary from zero to several tenths of $\mu \mathrm{eVs}$. All HQVs that we observed in this sample were at regions where the splitting was less than our experimental resolution $(\approx 20 \mu \mathrm{eV})$. 
The sample we studied is the same CdTe/ $\mathrm{CdMgTe}$ microcavity that was used in our previous experiments cooled down to $\sim 10 \mathrm{~K}$ by a liquid helium flow cryostat (4). We used continuouswave monomode nonresonant optical excitation. Detection was performed by means of the modified Michelson interferometer in the mirrorretroflector configuration with active stabilization (4) completed by a lambda quarter and a Wollaston prism. This allowed for polarizationresolved interferometry in $\sigma^{+}$and $\sigma^{-}$polarizations simultaneously, which facilitated the identification of half vortices (21). All spectral studies were performed using a monochromator with $\approx 20 \mu \mathrm{eV}$ resolution. The output of the interferometer could be sent to the entrance slits of the spectrometer through a polarizer, allowing for spectrally and polarization-resolved interferometry images to be acquired. The HQV were observed only at the excitation powers exceeding the condensation threshold. Once a good candidate was found, we performed a number of preliminary "test experiments" to verify unambiguously the persistence of the vortex for all possible detection configurations. The two most reliable tests were to change the overlap conditions at the output of the interferometer by shifting the mirror arm image with respect to one reflected from the retroreflector and to rotate by $\pi$ the orientation of the fringes, making sure that for all orientations the singularity of the vortex is always clearly observable (4). We took care to verify the mutual coherence of the two cross-circular polarization components by means of polarization mixing interferometry in order to eliminate the possibility of having two independent condensates in the two polarizations. In all cases, we observed excellent mutual coherence properties with good contrast in the interference fringes between the two circular polarization components (21). The appearance of half vortices was quite rare; that is, one out of six regions with no polarization splitting was exhibiting a HQV.

Once the HQV was identified, the interferometric image was being sent on the entrance slits of the spectrometer. Then we performed an optical tomography experiment (25) for $\sigma^{+}$and $\sigma^{-}$ polarized images, which provided us with the full set of polarization-resolved interferograms in real space for all frequencies within the observable spectral window. Figure 1, A and B, shows the reconstructed interferogram coming from the frequency of the polariton condensate for the polarizations $\sigma^{+}$and $\sigma^{-}$, on which we have added a red circle centered at the vortex core to help the reader locate the singularity. The singularity (forklike dislocation) is clearly visible for the $\sigma^{+}$ polarization, whereas on the same position in real space for the $\sigma^{-}$polarization we observe straight fringes. The interference patterns gave us access to the phase of the coherent polariton fluid. To extract the phase, we assumed that the reference field coming from a region of the condensate without a vortex has a flat phase profile. Figure 1, $\mathrm{C}$ and $\mathrm{D}$, shows the phase of the polariton fluid in real space calculated from the interferograms. The phase has distinguishable characteristics only where there is enough signal intensity, whereas at the regions with no signal we get a noisy phase with no distinguishable features. The position of the HQV in the phase map is highlighted by circles. In $\sigma^{+}$polarization, the phase rotates by $2 \pi$ as one goes around the core. This behavior of the phase is clearly seen within an area of a few micrometers' size. In the same region for the $\sigma$ polarization, there are no observable singularities and the phase is homogeneous. Figure 1, E and F, shows the phase as a function of the azimuthal angle as one goes around the core along the circles of different radii (shown by color). For the radius of $1 \mu \mathrm{m}$, the phase changes monotonously in $\sigma^{+}$polarization, decreasing by $2 \pi$ as one makes a full round. Contrary to this, for the same radius in $\sigma^{-}$polarization, we observe a quasi-flat phase profile indicating the lack of any singularity. For larger radii, the phase dependence on the azimuthal angle becomes strongly nonlinear, whereas the total phase shift as one goes around the core remains $-2 \pi$ for one and 0 for the other polarization. Distortion of the phase profile at the large radii may be indicative of the existence of nearby regions with substantial vorticity but can also be indicative of formation of a string.

We note that the specific HQV shown in Fig. 1 is characterized by the winding numbers $(k, m)=\left(+\frac{1}{2},-\frac{1}{2}\right)$, while we have observed also the three remaining types of HQVs in different locations on the sample (21). On the basis of measurements we have done, we believe that four possible types of HQV are realized with approximately the same probability in our sample.

Using the same tomographic technique of spectrally resolved real space imaging as before, we then probed only the density of polaritons in the condensate state (Fig. 2, A and B). Locating the vortex in real space and looking at the density close to its core, we observed that a local minimum for one polarization coincides with a maximum for the other one, as Fig. 2, C and D shows. The widths of these minima/maxima coincide with the theoretical vortex core size $a$. This behavior is another signature of HQVs, as one can see from Eq. 3. The theory (12) predicted that at the center of the HQV the condensate should be fully circularly polarized, and this is exactly what we observe in Fig. 2, C and D).

The HQV we observed here are pinned by the disorder to specific locations on the sample. This is confirmed by the behavior of the interferometric images as a function of the pumping power. Increasing the excitation intensity, we modify the effective disorder potential acting upon the polariton condensate by changing the polaritonpolariton repulsion strength. When the pumping is strong, we screen the disorder potential so that HQV get unpinned and disappear from the interferometry image of a specific spot on the sample. This is what we observed at the excitation power exceeding the threshold pumping by a factor of 4.5. Above this power, the forklike dislocation in $\sigma^{+}$polarization disappears $(21)$.
The stationary disorder fixes the winding numbers of the pinned vortices, so that repeating the experiment we find HQVs with the same winding numbers in the same locations. This is also true for the integer vortices. Handedness of each pinned vortex is dependent on the direction of polariton fluxes propagating in the disorder landscape during formation of the condensate, as the modeling based on the Gross-Pitaevskii equation showed $(4,26)$.

This experimental work provides direct evidence of half-quantum vortices in a spinor condensate, by means of polarization-resolved interferometry, phase imaging, and spectrally resolved real space density imaging.

\section{References and Notes}

1. L. P. Pitaevskii, S. Stringari, Bose-Einstein Condensation (Clarendon Press, Oxford, 2003).

2. R. J. Donnelly, Quantized Vortices in Helium II (Cambridge Univ. Press, Cambridge, England, 1991).

3. M. R. Matthews et al., Phys. Rev. Lett. 83, 2498 (1999).

4. K. G. Lagoudakis et al., Nat. Physics 4, 706 (2008).

5. M. M. Salomaa, G. E. Volovik, Rev. Mod. Phys. 59, 533 (1987)

6. G. E. Volovik, The Universe in a Helium Droplet (Clarendon Press, Oxford, 2003).

7. I. A. Luk'yanchuk, M. E. Zhitomirsky, Supercond. Rev 1, 207 (1995)

8. M. Kato, Y. Niwa, K. Maki, J. Phys. Conf. Ser. 150, 052103 (2009)

9. M. Yamashita et al., Phys. Rev. Lett. 101, 025302 (2008).

10. ]. R. Kirtley et al., Phys. Rev. Lett. 76, 1336 (1996).

11. C. C. Tsuei, ]. R. Kirtley, Physica C 367, 1 (2002).

12. Y. G. Rubo, Phys. Rev. Lett. 99, 106401 (2007).

13. A. V. Kavokin, J. J. Baumberg, G. Malpuech, F. P. Laussy, Microcavities (Oxford Univ. Press, Oxford 2007).

14. J. Kasprzak et al., Nature 443, 409 (2006).

15. R. Balili et al., Science 316, 1007 (2007).

16. S. Utsunomiya et al., Nat. Physics 4, 700 (2008).

17. A. Amo et al., Nature 457, 291 (2009).

18. M. R. Matthews et al., Phys. Rev. Lett. 83, 2498 (1999).

19. S. M. Girvin, A. H. MacDonald, in Perspectives in Quantum Hall Effects, S. Das Sarma, A. Pinczuk, Eds. (Wiley, New York, 1997), chap. 5.

20. H. A. Fertig, Phys. Rev. Lett. 89, 035703 (2002).

21. Materials and methods are available as supporting material on Science Online.

22. G. Panzarini et al., Phys. Rev. B 59, 5082 (1999).

23. J. Kasprzak et al., Phys. Rev. B 75, 045326 (2007).

24. Ł. Kłopotowski et al., Sol. St. Com. 139, 511 (2006).

25. G. Nardin et al., Appl. Phys. Lett. 94, 181103 (2009).

26. T. C. H. Liew, Y. G. Rubo, A. V. Kavokin, Phys. Rev. Lett. 101, 187401 (2008).

27. We thank Le Si Dang for crucial remarks and detailed proofreading of this manuscript. We also thank T. C. H. Liew, M. Toledo Solano, M. Wouters, B. Pietka, Y. Leger, M. Richard, A. Baas, G. Nardin, D. N. Krizhanovskii, D. Sanvitto, P. G. Lagoudakis, and V. Savona for enlightening discussions. K.G.L. and B.D.-P. thank the Quantum Photonics-National Centre of Competence in Research for financial support through the Swiss National Science Foundation. A.V.K. and Y.G.R. thank the Engineering and Physical Sciences Research Council and the Dirección General de Asuntos del Personal Académico de la Universidad Nacional Autónoma de México for support.

\section{Supporting Online Material}

www.sciencemag.org/cgi/content/full/326/5955/974/DC1 Materials and Methods

Figs. $\mathrm{S} 1$ to $\mathrm{S} 9$

References

19 June 2009; accepted 8 September 2009 10.1126/science. 1177980 